Research Paper

\title{
Microbleeds and Silent Brain Infarctions Are Differently Associated with Cognitive Dysfunction in Patients with Advanced Periventricular Leukoaraiosis
}

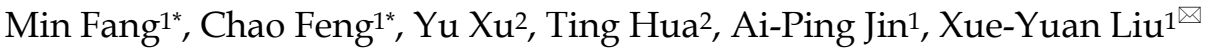 \\ 1. Department of Neurology, Shanghai Tenth People's Hospital of Tongji University, Shanghai, China; \\ 2. Department of Radiology, Shanghai Tenth People's Hospital of Tongji University, Shanghai, China. \\ *Both Min Fang and Chao Feng contributed equally to this paper.
}

$\triangle$ Corresponding author: Xue-Yuan Liu, Tel.: +86-21-66302582, E-mail: liuxy@tongji.edu.cn; and Ai-Ping Jin, Tel.: +86-21-66302551, E-mail: liu781234@sina.com, Department of Neurology, Shanghai Tenth People's Hospital of Tongii University, Middle Yanchang Rd. 301\#, Zhabei District, Shanghai, China.

(c) Ivyspring International Publisher. This is an open-access article distributed under the terms of the Creative Commons License (http://creativecommons.org/ licenses/by-nc-nd/3.0/). Reproduction is permitted for personal, noncommercial use, provided that the article is in whole, unmodified, and properly cited.

Received: 2013.04.09; Accepted: 2013.07.23; Published: 2013.08.08

\begin{abstract}
Background: Leukoaraiosis, microbleeds, and silent brain infarctions are phenotypes of small vessel disease. Leukoaraiosis is the most prevalent, and advanced periventricular leukoaraiosis is regarded as a strong predictor of cognitive dysfunction. Microbleeds and silent brain infarctions sometimes coexist with leukoaraiosis. This study aims to analyze the effects of microbleeds and silent brain infarctions on cognitive function of patients with advanced periventricular leukoaraiosis.

Methods: 227 patients with advanced periventricular leukoaraiosis were divided into control, MB, $\mathrm{SBI}$, and MB\&SBI groups. The presence and locations of microbleeds and silent brain infarctions were evaluated. Mini-Mental State Examination, Montreal Cognitive Assessment, Clock Drawing Test and Verbal Fluency Test were performed. Chi-square test and ANOVA to compare the characteristics of four groups, multiple linear regressions to identify the risk factors for cognitive dysfunction.

Results: The scores in all four tests were lower in the MB and MB\&SBI groups while only the scores in Clock Drawing Test and Verbal Fluency Test were lower in the SBI group than in the control group. Age and the presence of microbleeds were independent risk factors for the lower scores in all four tests, whereas the presence of silent brain infarctions was the only independent risk factor for the lower scores in Clock Drawing Test and Verbal Fluency Test. Lobar microbleeds had the most significant effect on cognitive function.

Conclusion: Microbleeds and silent brain infarctions were associated differently with cognitive impairment of patients with advanced periventricular leukoaraiosis. The effect of lobar microbleeds was the most significant.
\end{abstract}

Key words: small vessel disease, leukoaraiosis, silent brain infarctions, microbleeds, cognitive dysfunction.

\section{Introduction}

Leukoaraiosis, microbleeds, and silent brain infarctions are different phenotypes of small vessel disease caused by different kinds of arteriole degen- eration ${ }^{[1,2]}$. Leukoaraiosis is the most common phenotype of small vessel disease and has a prevalence of over $60 \%$ among elderly ${ }^{[3]}$. Advanced periventricular 
leukoaraiosis is believed to indicate a severe stage of small vessel disease and predict cognitive dysfunction or vascular dementia[3-6]. Other phenotypes of small vessel disease such as microbleeds and silent brain infarctions are usually found coexisting with advanced leukoaraiosis[7-11] and they might also be associated with cognitive dysfunction ${ }^{[10,12-14]}$. However, it remains unclear whether the coexisting microbleeds and silent brain infarctions will deteriorate the cognitive function for patients with advanced periventricular leukoaraiosis, and whether microbleeds and silent brain infarctions have different impact on cognitive function considering they might be different in specific pathogenesis. In this study, we enrolled a group of patients with advanced periventricular leukoaraiosis and sought to answer the questions above.

\section{Subjects and Methods}

\section{General information}

In this paper, a cross-sectional study was performed based on the patients attending to the Department of Neurology in 10 th People's Hospital in Shanghai from January 2012 to September 2012, mainly with complaints of non-specific symptoms such as headache, dizziness, vertigo, and dysmnesia. The patients were enrolled according to the following criteria: 1) three years or longer education, with basic reading ability and will to give consent to this study; 2) no history of stroke, simple Alzheimer's Disease, dementia with Lewy bodies, schizophrenia, or other diseases that might cause the decline in cognitive function and behavior competence; or newly diagnosis of above mentioned diseases during this study; 3 ) no claustrophobia or metallic implants; 4) periventricular leukoaraiosis of grades 2 to 3 identified through magnetic resonance imaging (MRI) according to Fazekas' scale ${ }^{[15]}$.

Silent brain infarctions were counted on T1- and T2-weighted images and fluid-attenuatedinversion-recovery (FLAIR) images. Susceptibility weighted images (SWI) were further obtained to evaluate the presence and number of microbleeds. According to the presence of silent brain infarctions and microbleeds, 227 patients enrolled were further divided into four groups: control (91 patients without silent brain infarctions and microbleeds), MB (41 patients with microbleeds and without silent brain infarctions), SBI (46 patients with silent brain infarctions and without microbleeds), and MB\&SBI (49 patients with both microbleeds and silent brain infarctions) groups.

The following pieces of information were collected: age, sex, education years, the presence of hypertension and diabetes, smoking history, and blood test including blood glucose and lipid. Mini-Mental State Examination (MMSE) and Montreal Cognitive Assessment (MoCA) were used to test the global cognitive function. Clock Drawing Test ${ }^{[16-18]}$ and Verbal Fluency Test ${ }^{[19,20]}$ were performed to evaluate the executive function which was proved to be a cognitive domain impaired by leukoaraiosis most frequently. The result of Clock Drawing Test was scored from 0 to 4 according to the integrity of the clock drawn by the patients. The result of Verbal Fluency Test was scored based on the amount of different products sold in a supermarket by the patients in $1 \mathrm{~min}$. Clock Drawing Test and Verbal Fluency Test were both used to evaluate executive function ${ }^{[21,22]}$. All the procedures were approved by the Ethics Committee of $10^{\text {th }}$ People's Hospital.

\section{MR protocol}

The subjects were imaged using a 3.0T MR scanner (Siemens 3.0T Magnetom Verio, Erlangen, Germany) with a standard eight-channel phased-array head coil. The MRI imaging protocol consists of T1-weighted images (repetition time/echo time $=2000 / 9$ ), FLAIR (repetition time/echo time $=$ $8500 / 94$ ), and diffusion-weighted imaging (repetition time $/$ echo time $=6000 / 94$ ) on the axial plane; T2-weighted images (repetition time/echo time $=$ 4540/96) on the sagittal plane with a thickness of 5.5 $\mathrm{mm}$; and SWI (repetition time/echo time $=27 / 20$ ) on the axial plane with a thickness of $1.5 \mathrm{~mm}$.

\section{MR image review and criteria}

All MR images were analyzed by two radiologists blinded to the clinical information. Discrepancies in the presence, numbers, locations of silent brain infarctions and microbleeds and the scores of leukoaraiosis between the two readers were resolved using a visual consensus.

Leukoaraiosis was defined as focal or confluent hyperintensities in the deep or periventricular area on FLAIR images ${ }^{[3]}$. Lesions were defined as periventricular leukoaraiosis when their largest diameters are adjacent to the ventricles ${ }^{[3]}$. Grade 2-3 according to Fazekas' scale[15] was regarded as advanced leukoaraiosis. Silent brain infarction was defined as a focal cavitated lesion $3 \mathrm{~mm}$ to $15 \mathrm{~mm}$ in diameter, with hypointensity on T1-weighted images, hyperintensity on T2-weighted images, without corresponding stroke history ${ }^{[10]}$. The presence and numbers of silent brain infarctions in basal ganglia, thalamus, corona radiata and brain stem were evaluated respectively. Microbleeds were defined as homogenous round areas of signal loss with diameters less than $10 \mathrm{~mm}$ on SWI images ${ }^{[23]}$. The presence and numbers of lobar, deep, and infratentorial Mi- 
crobleeds were evaluated. Hypointense lesions within the subarachnoid space, basal ganglia mineralization, and other lesions or structures with similar signals were excluded during the microbleeds' evaluation ${ }^{[23}$, 24].

\section{Statistical Analysis}

All data were analyzed using SAS 9.2. ANOVA and Chi-square test were used to compare the baseline characteristics, including age, sex, vascular risk factors, and scores in the four neuropsychological tests among the groups. The Least Significance Difference Test was used for the multiple comparisons of the scores in the four tests. Multiple linear regression models were then constructed to identify the independent risk factors for the low scores in the four tests, with age, sex, education years, smoking history, and the presence of hypertension, diabetes, dyslipidemia, microbleeds, and silent brain infarctions added into the models. $\mathrm{P}<0.05$ was considered to indicate statistical difference, whereas $\mathrm{P}<0.01$ was considered to indicate significant statistical difference.

\section{Results}

\section{Baseline characteristics and results of the dif- ferent neuropsychological tests}

No statistical differences in age, sex, and vascular risk factors were found among the four groups. By contrast, significant differences in the scores in all four neuropsychological tests were found among four groups. Multiple comparisons of the four scales were further carried out among the groups. The results showed that the scores in all four tests were lower in the MB and MB\&SBI groups than in the control group (all $\mathrm{P}<0.05)$, whereas only the scores in Clock Drawing Test and Verbal Fluency Test were lower in the SBI group than in the control group (both $\mathrm{P}<0.05$ ). Multiple comparisons showed no statistical differences in the scores in all tests among the MB, SBI, and MB\&SBI groups (all $\mathrm{P}>0.05$ ). However, a downtrend can be observed from the control group to the MB\&SBI group for all tests, and the scores in the four tests are the lowest in the MB\&SBI group. The details are shown in Table 1. Examples of leukoaraiosis, silent brain infarctions and microbleeds were shown in Figure 1.

Table I. Baseline characteristics and results of the neuropsychological tests.

\begin{tabular}{llllll}
\hline & \multicolumn{1}{c}{ Control $(\mathrm{n}=91)$} & SBI $(\mathrm{n}=46)$ & MB $(\mathrm{n}=41)$ & MB\&SBI $(\mathrm{n}=49)$ & $\mathrm{P}$ \\
\hline Age (years) & $71.4 \pm 5.9$ & $70.9 \pm 6.4$ & $70.6 \pm 5.2$ & $72.1 \pm 5.0$ & $>0.05$ \\
Male, $\mathrm{n}(\%)$ & $47(51.7)$ & $20(43.5)$ & $24(58.5)$ & $26(53.1)$ & $>0.05$ \\
Smoking, $\mathrm{n}(\%)$ & $21(23.1)$ & $9(19.6)$ & $9(22.0)$ & $12(24.5)$ & $>0.05$ \\
Hypertension, $\mathrm{n}(\%)$ & $68(74.7)$ & $38(82.6)$ & $33(80.5)$ & $40(81.6)$ & $>0.05$ \\
Diabetes, $\mathrm{n}(\%)$ & $24(26.4)$ & $10(21.7)$ & $11(26.8)$ & $24.5)$ & $>0.05$ \\
Dyslipidemia, $\mathrm{n}(\%)$ & $47(51.7)$ & $24(52.2)$ & $24(58.5)$ & $27(55.1)$ & $>0.05$ \\
Education (years) & $8.14 \pm 2.12$ & $8.11 \pm 2.32$ & $8.24 \pm 1.85$ & $8.20 \pm 2.31$ & $>0.05$ \\
MMSE & $26.6 \pm 2.0$ & $25.7 \pm 2.2$ & $25.1 \pm 3.7$ & $25.0 \pm 3.1$ & $\mathbf{0 . 0 0 2}$ \\
MoCA & $22.9 \pm 2.2$ & $21.9 \pm 2.3$ & $21.0 \pm 3.6$ & $21.0 \pm 3.5$ & $<0.001$ \\
CDT & $3.24 \pm 0.75$ & $2.87 \pm 0.61$ & $2.76 \pm 0.86$ & $2.71 \pm 0.79$ & $<0.001$ \\
VFT & $23.0 \pm 4.5$ & $21.4 \pm 4.8$ & $20.6 \pm 5.3$ & $19.5 \pm 4.7$ & $<0.001$ \\
\hline
\end{tabular}

$\mathrm{CDT}=$ Clock Drawing Test, $\mathrm{VFT}=$ Verbal Fluency Test .

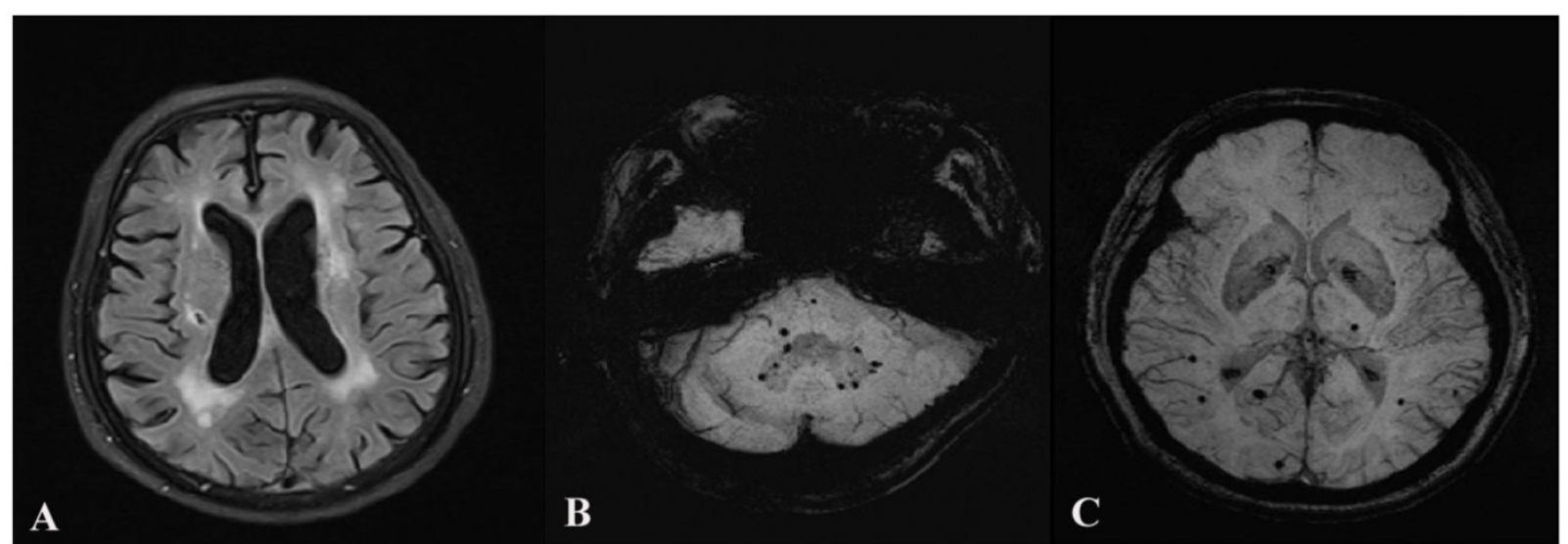

Fig I. Advanced periventricular leukoaraiosis, silent brain infarction and microbleeds. Leukoaraiosis and silent brain infarction are shown on Image A. Lobar, deep and infratentorial microbleeds are shown on Image B and C. 


\section{Risk factors of cognitive impairment}

Multiple linear models were constructed to identify the independent risk factors of cognitive impairment. Age, sex, education years, smoking history, and the presence of hypertension, diabetes, dyslipidemia, microbleeds, and silent brain infarctions were added into the four models. The results showed that age and presence of microbleeds were the independent risk factors for the lower scores in all four tests, whereas the presence of silent brain infarctions was the only independent risk factor for the lower scores in Clock Drawing Test and Verbal Fluency Test. The effect of microbleeds on cognitive impairment was more evident than that of silent brain infarctions. The details of the positive results are listed in Table 2.

\section{Effects of microbleeds in different locations on cognitive function}

The effect of microbleeds in different locations on cognitive function was further analyzed using multiple linear regressions. The numbers of lobar, deep and infratentorial microbleeds were added into the models together with age, sex, education years, smoking history, and the presence of hypertension, diabetes, dyslipidemia, and silent brain infarctions. The results showed that lobar microbleeds had the most significant effect on cognitive function. The details are shown in Table 3.

\section{Effects of silent brain infarctions in different locations on cognitive function}

The effect of silent brain infarctions in different locations on cognitive function was further analyzed with multiple linear regressions too. The numbers of silent brain infarctions in basal ganglia, thalamus, corona radiata and brain stem were added into the four models with age, sex, education years, smoking history, and the presence of hypertension, diabetes, dyslipidemia and microbleeds. The results showed no obvious tendency that silent brain infarctions in some special location had more significant effect on cognitive function than those in other locations. The association between cognitive dysfunction and silent brain infarctions in different locations was weakened after they were divided into subgroups. The details are shown in Table 4.

Table 2. Independent risk factors for cognitive impairment.

\begin{tabular}{lllllllll}
\hline & MMSE & & MoCA & & CDT & \multicolumn{2}{l}{ VFT } \\
\hline & $\mathrm{t}$ & $\mathrm{P}$ & $\mathrm{t}$ & $\mathrm{P}$ & $\mathrm{t}$ & $\mathrm{P}$ & $\mathrm{t}$ & $\mathrm{P}$ \\
\hline Age & -7.059 & $<0.001$ & -6.621 & $<0.001$ & -5.181 & $<0.001$ & -5.684 & $<0.001$ \\
Education & - & - & - & - & - & - & 1.982 & 0.049 \\
Diabetes & - & - & - & - & -2.353 & 0.019 & - & - \\
Microbleeds & -3.243 & 0.001 & -3.956 & $<0.001$ & -3.219 & 0.001 & -3.345 & 0.001 \\
Silent brain infarctions & - & - & - & - & -2.231 & 0.027 & -2.160 & 0.032 \\
\hline
\end{tabular}

$\mathrm{CDT}=$ Clock Drawing Test, $\mathrm{VFT}=$ Verbal Fluency Test.

Table 3. Effects of microbleeds in different locations on cognitive function.

\begin{tabular}{|c|c|c|c|c|c|c|c|c|}
\hline & \multicolumn{2}{|l|}{ MMSE } & \multicolumn{2}{|l|}{ MoCA } & \multicolumn{2}{|l|}{ CDT } & \multicolumn{2}{|l|}{ VFT } \\
\hline & $\mathrm{t}$ & $\mathrm{P}$ & $\mathrm{t}$ & $\mathrm{P}$ & $\mathrm{t}$ & $\mathrm{P}$ & $\mathrm{t}$ & $\mathrm{P}$ \\
\hline Age & -7.590 & $<0.001$ & -7.016 & $<0.001$ & -4.965 & $<0.001$ & -5.529 & $<0.001$ \\
\hline Education & 2.216 & 0.035 & - & - & - & - & - & - \\
\hline Diabetes & - & - & - & - & -2.273 & 0.024 & - & - \\
\hline Silent brain infarctions & - & - & - & - & -2.459 & 0.015 & -2.519 & 0.013 \\
\hline \multicolumn{9}{|c|}{ Microbleeds in different locations } \\
\hline Lobar & -8.000 & $<0.001$ & -7.761 & $<0.001$ & -2.994 & 0.003 & -4.971 & $<0.001$ \\
\hline Deep & - & - & - & - & - & - & - & - \\
\hline Infratentorial & - & - & - & - & -2.058 & 0.041 & - & - \\
\hline
\end{tabular}

CDT $=$ Clock Drawing Test, VFT $=$ Verbal Fluency Test . 
Table 4. Effects of silent brain infarctions in different locations on cognitive function.

\begin{tabular}{|c|c|c|c|c|c|c|c|c|}
\hline & \multicolumn{2}{|l|}{ MMSE } & \multicolumn{2}{|l|}{ MoCA } & \multicolumn{2}{|l|}{ CDT } & \multicolumn{2}{|l|}{ VFT } \\
\hline & $\mathrm{t}$ & $\mathrm{P}$ & $\mathrm{t}$ & $\mathrm{P}$ & $\mathrm{t}$ & $\mathrm{P}$ & $\mathrm{t}$ & $\mathrm{P}$ \\
\hline Age & -6.817 & $<0.001$ & -6.386 & $<0.001$ & -4.854 & $<0.001$ & -5.414 & $<0.001$ \\
\hline Diabetes & - & - & - & - & -2.133 & 0.034 & - & - \\
\hline Microbleeds & -3.078 & 0.002 & -3.839 & $<0.001$ & -3.160 & 0.002 & -3.273 & 0.001 \\
\hline \multicolumn{9}{|c|}{ Silent brain infarctions in different locations } \\
\hline Basal ganglia & - & - & -1.980 & 0.049 & - & - & -2.002 & 0.047 \\
\hline Thalamus & - & - & - & - & - & - & - & - \\
\hline Corona radiata & - & - & - & - & - & - & -2.120 & 0.035 \\
\hline Brain stem & - & - & - & - & - & - & - & - \\
\hline
\end{tabular}

CDT $=$ Clock Drawing Test, VFT $=$ Verbal Fluency Test.

\section{Discussion}

This study showed that both microbleeds and silent brain infarctions might exacerbate cognitive impairment for patients with advanced periventricular leukoaraiosis; however, their effects differed to some extent.

The strengths of this study were the use of multiple groups, comparison between microbleeds and silent brain infarctions as different phenotypes of small vessel disease, different neuropsychological tests for the evaluation of different cognitive domains, and the utilization of SWI which was proven better than T2-GRE sequences for the identification of microbleeds. In addition, the roles of microbleeds and silent brain infarctions were further analyzed according to different locations. The main limitations were the hospital-based sample, limited sample size, and the non-prospective study design that might restrict the significance of our results.

Previous studies proved that leukoaraiosis, particularly advanced periventricular leukoaraiosis, is associated with the decline in cognitive function ${ }^{[3-6]}$. Most of these studies were based on MMSE, which is not sensitive enough to detect subtle changes in cognitive function [25]. In this study, MoCA and MMSE were used together for the evaluation of global cognitive function. We found that the average score of MoCA was only 22.9 for control group, lower than that of MMSE. This result verified the association between leukoaraiosis and cognitive impairment ${ }^{[6]}$ and the higher sensitivity of MoCA compared with MMSE[25]. Advanced leukoaraiosis sometimes coexists with other phenotypes of small vessel disease such as microbleeds and silent brain infarctions. In this study, we proved that the decline in cognitive function might overlap and become even steeper when different phenotypes of small vessel disease coexist. Moreover, the effects of microbleeds and silent brain infarctions on cognitive function and cognitive domains were different to some extent.
In this study, microbleeds, particularly lobar microbleeds, showed a more significant negative effect on cognitive impairment than silent brain infarctions. Previous studies proved that microbleeds are associated with the decline in different functional domains, including information processing speed, executive function, and motor speed[12, 26-28]. For patients with advanced periventricular leukoaraiosis, which is an indicator of severe underlying hypertensive small vessel disease and white matter degeneration, the coexistence of microbleeds could significantly deteriorate cognitive function. However, this trend of deterioration was not that evident for silent brain infarctions, and was mainly restricted to executive function. Their different effect on cognitive function could be attributed to the different underlying vascular diseases and different locations.

For silent brain infarctions, they were widely believed to be related to arteriolosclerosis caused by hypertension, and most were located in perforating artery territories such as corona radiata and basal ganglia (i.e., deep in the brain). This characteristic is similar to leukoaraiosis in both pathogenesis and locations $[1,3,10,29]$. Thus, the steeper decline in cognitive function reflected the overlapping of the severity of a similar small vessel disease. The limited effect of silent brain infarctions on global cognitive function could also be explained by the deep distribution, that is, the deep area in the brain that was less closely associated with global cognitive function and more closely associated with executive function. This finding was also proven by previous studies ${ }^{[14,30]}$. Unlike microbleeds, most silent brain infarctions were concentrated in deep regions of brains, with the same pathogenesis for them in basal ganglia, corona radiata or thalamus. Therefore, after silent brain infarctions were divided into subgroups, the association between cognitive dysfunction and silent brain infarctions was weakened, with no obvious tendency that silent brain infarctions in some special location had more significant 
effect on cognitive function than those in other locations.

Meanwhile, the locations and pathogenesis of microbleeds were variable. Some microbleeds identified in this study were located in lobes, and these lobar microbleeds were proven to have the most significant effect in worsening cognitive function. By contrast, the association between cognitive impairment and deep or infratentorial microbleeds was not that robust, more like that between cognitive impairment and silent brain infarctions. The significant role of lobar microbleeds could partly be explained by the strategic importance of lobes in cognitive function [28]. Furthermore, these microbleeds might also be related to different pathogenesis. Most studies believed that strictly lobar microbleeds might be related to cerebral amyloid angiopathy[31,32], which refers to the deposition of $\beta$-amyloid. On the other hand, similarly to silent brain infarctions, deep and infratentorial microbleeds were proven to be caused by hypertensive arteriolosclerosis[33, 34]. Although cerebral amyloid angiopathy and arteriolosclerosis are both attributed to the category of small vessel disease [1], their roles in cognitive dysfunction might be different, with the former more significant and better-known because of Alzheimer's Disease. Thus for patients with both advanced leukoaraiosis and lobar microbleeds, the decline in cognitive function was not attributed to the simple overlapping of similar vascular diseases but to the combination of different pathogenesis in different locations, similarly to that of mixed dementia with part of the pathogenesis resembling Alzheimer's Disease.

In conclusion, we found that both silent brain infarctions and microbleeds might exacerbate cognitive dysfunction. The effect of microbleeds seemed to be more significant than silent brain infarctions. This significant effect was mostly contributed by lobar microbleeds. The results of this study proved that vascular dementia and its early stage, i.e., vascular cognitive dysfunction for non-stroke patients might be the consequence of various subtypes of small vessel disease including leukoaraiosis, microbleeds and silent brain infarctions; part of it more resembled mixed dementia or cognitive dysfunction. The results also suggested that, the prevention of microbleeds and silent brain infarctions especially the former should not be neglected as a possible target for the intervention of cognitive dysfunction.

\section{Acknowledgement}

This study was supported by grants from the National Natural Science Foundation of China (No.81000492, No.30971029, No.81171163).

\section{Competing Interests}

The authors have declared that no competing interest exists.

\section{References}

1. Moran C, Phan TG, Srikanth VK. Cerebral small vessel disease: A review of clinical, radiological, and histopathological phenotypes. International Journal of Stroke. 2012;7:36-46.

2. Pantoni L. Cerebral small vessel disease: From pathogenesis and clinical characteristics to therapeutic challenges. Lancet Neurol. 2010;9:689-701.

3. Xiong YY, Mok V. Age-related white matter changes. Journal of Aging Research. 2011;2011:1-13.

4. Xiong Y, Mok V, Wong A, Chen X, Chu WC, Fan Y, Soo Y, Wong KS. The age-related white matter changes scale correlates with cognitive impairment. Eur J Neurol. 2010;17:1451-1456.

5. Debette S, Markus HS. The clinical importance of white matter hyperintensities on brain magnetic resonance imaging: Systematic review and meta-analysis. Bmj. 2010;341:c3666.

6. de Groot JC, de Leeuw FE, Oudkerk M, Hofman A, Jolles J, Breteler MM. Cerebral white matter lesions and subjective cognitive dysfunction: The rotterdam scan study. Neurology. 2001;56:1539-1545.

7. Chowdhury MH, Nagai A, Bokura H, Nakamura E, Kobayashi S, Yamaguchi S. Age-related changes in white matter lesions, hippocampal atrophy, and cerebral microbleeds in healthy subjects without major cerebrovascular risk factors. Journal of Stroke and Cerebrovascular Diseases. 2011;20:302-309.

8. Fan YH, Mok VCT, Lam WWM, Hui ACF, Wong KS. Cerebral microbleeds and white matter changes in patients hospitalized with lacunar infarcts. Journal of Neurology. 2004;251:537-541.

9. Fisher M. Cerebral microbleeds and white matter disease: Separated at birth? European Journal of Neurology. 2012;19:2-3.

10. Vermeer SE, Longstreth WT, Koudstaal PJ. Silent brain infarcts: A systematic review. The Lancet Neurology. 2007;6:611-619.

11. Geerlings MI, Appelman APA, Vincken KL, Mali WPTM. Association of white matter lesions and lacunar infarcts with executive functioning: The smart-mr study. American Journal of Epidemiology. 2009;170:1147-1155.

12. M.M.F. Poels MAI, A. van der Lugt, A. Hofman, W.J. Niessen, G.P. Krestin, M.M.B. Breteler, M.W. Vernooij. Cerebral microbleeds associated with worse cognitive function: The rotterdam scan study. Neurology. 2012;78:326-333.

13. Koga H, Takashima Y, Murakawa R, Uchino A, Yuzuriha T, Yao H. Cognitive consequences of multiple lacunes and leukoaraiosis as vascular cognitive impairment in community-dwelling elderly individuals. Journal of Stroke and Cerebrovascular Diseases. 2009;18:32-37.

14. van Dijk EJ, Prins ND, Vrooman HA, Hofman A, Koudstaal PJ, Breteler MMB. Progression of cerebral small vessel disease in relation to risk factors and cognitive consequences: Rotterdam scan study. Stroke. 2008;39:2712-2719.

15. Franz Fazekas JBC, Abass Alavi, Howard I. Hurtig, Robert A. Zimmerman. Mr signal abnormalities at $1.5 \mathrm{t}$ in alzheimer's dementia and normal aging. AJR. 1987;149:351-356.

16. Korner EA, Lauritzen L, Nilsson FM, Lolk A, Christensen P. Simple scoring of the clock-drawing test for dementia screening. Dan Med J. 2012;59:A4365.

17. Watson YI, Arfken CL, Birge SJ. Clock completion: An objective screening test for dementia. I Am Geriatr Soc. 1993;41:1235-1240.

18. Kim YS, Lee KM, Choi BH, Sohn EH, Lee AY. Relation between the clock drawing test (cdt) and structural changes of brain in dementia. Arch Gerontol Geriatr. 2009;48:218-221.

19. Mok EH, Lam LC, Chiu HF. Category verbal fluency test performance in chinese elderly with alzheimer's disease. Dement Geriatr Cogn Disord. 2004;18:120-124.

20. Wysokinski A, Zboralski K, Orzechowska A, Galecki P, Florkowski A, Talarowska M. Normalization of the verbal fluency test on the basis of results for healthy subjects, patients with schizophrenia, patients with organic lesions of the chronic nervous system and patients with type 1 and 2 diabetes. Arch Med Sci. 2010;6:438-446.

21. Brooks BL, Iverson GL, Lanting SC, Horton AM, Reynolds CR. Improving test interpretation for detecting executive dysfunction in adults and older adults: Prevalence of low scores on the test of verbal conceptualization and fluency. Appl Neuropsychol. 2012;19:61-70.

22. Woo BK, Rice VA, Legendre SA, Salmon DP, Jeste DV, Sewell DD. The clock drawing test as a measure of executive dysfunction in elderly depressed patients. J Geriatr Psychiatry Neurol. 2004;17:190-194.

23. Koennecke HC. Cerebral microbleeds on mri: Prevalence, associations, and potential clinical implications. Neurology. 2006;66:165-171. 
24. Shoamanesh A, Kwok CS, Benavente O. Cerebral microbleeds: Histopathological correlation of neuroimaging. Cerebrovascular Diseases. 2011;32:528-534.

25. Dong Y, Sharma VK, Chan BP, Venketasubramanian N, Teoh HL, Seet $\mathrm{RC}$, Tanicala S, Chan YH, Chen C. The montreal cognitive assessment (moca) is superior to the mini-mental state examination (mmse) for the detection of vascular cognitive impairment after acute stroke. I Neurol Sci. 2010;299:15-18.

26. van Es AC, van der Grond J, de Craen AJ, Westendorp RG, Bollen EL, Blauw GJ, Greenberg SM, van Buchem MA. Cerebral microbleeds and cognitive functioning in the prosper study. Neurology. 2011;77:1446-1452.

27. Yakushiji Y, Nishiyama M, Yakushiji S, Hirotsu T, Uchino A, Nakajima J, Eriguchi M, Nanri Y, Hara M, Horikawa E, Kuroda Y. Brain microbleeds and global cognitive function in adults without neurological disorder. Stroke. 2008;39:3323-3328.

28. van Norden AG, van den Berg HA, de Laat KF, Gons RA, van Dijk EJ, de Leeuw FE. Frontal and temporal microbleeds are related to cognitive function: The radboud university nijmegen diffusion tensor and magnetic resonance cohort (run dmc) study. Stroke. 2011;42:3382-3386.

29. Vermeer SE. Prevalence and risk factors of silent brain infarcts in the population-based rotterdam scan study. Stroke. 2002;33:21-25.

30. Prins ND, van Dijk EJ, den Heijer T, Vermeer SE, Jolles J, Koudstaal PJ, Hofman A, Breteler MM. Cerebral small-vessel disease and decline in information processing speed, executive function and memory. Brain. 2005;128:2034-2041.

31. Dierksen GA, Skehan ME, Khan MA, Jeng J, Nandigam RN, Becker JA, Kumar A, Neal KL, Betensky RA, Frosch MP, Rosand J, Johnson KA, Viswanathan A, Salat DH, Greenberg SM. Spatial relation between microbleeds and amyloid deposits in amyloid angiopathy. Ann Neurol. 2010;68:545-548.

32. Mesker DJ, Poels MM, Ikram MA, Vernooij MW, Hofman A, Vrooman HA, van der Lugt A, Breteler MM. Lobar distribution of cerebral microbleeds: The rotterdam scan study. Arch Neurol. 2011;68:656-659.

33. Vernooij MW, van der Lugt A, Ikram MA, Wielopolski PA, Niessen WJ, Hofman A, Krestin GP, Breteler MM. Prevalence and risk factors of cerebral microbleeds: The rotterdam scan study. Neurology. 2008;70:1208-1214.

34. Greenberg SM, Nandigam RNK, Delgado P, Betensky RA, Rosand J, Viswanathan A, Frosch MP, Smith EE. Microbleeds versus macrobleeds: Evidence for distinct entities * supplemental materials and methods. Stroke. 2009;40:2382-2386. 